\title{
SPECIFIC IMMUNE MILK
}

In the second half of the twentieth century the use of antibiotics has transformed the practice of medicine. Many previously feared infectious diseases such as bacterial meningitis, pneumonia, puerperal fever, pyelonephritis, bacterial endocarditis and tuberculosis can now be treated and cured. The scope of surgery has also been greatly extended. Intensive care, transplantation and the management of severe trauma would be severely limited if antibiotics were not available to control secondary bacterial infection.

Currently used antibiotics are all based on chemicals produced naturally by bacteria and fungi. Competition between micro-organisms has led to the evolution of natural antibiotics, which give the secreting organism an advantage in its ecological niche, but the same evolutionary process has also led to the emergence of natural resistance. Increasing use of antibiotics in human therapy, and more controversially in animal husbandry, has led to the gradual emergence of resistant strains of bacteria in human disease. This problem is compounded by the fact that the wide range of antibiotics available is based on a relatively few chemical families and no new family has been discovered in the last decade. It seems possible that the development of new antibiotics will slow down as the emergence of antibiotic resistance accelerates and our ability to treat bacterial disease in the future could be seriously compromised.

Micro-organisms fight other micro-organisms using antibiotics. Mammals, however, use a rather more sophisticated system for fighting micro-organisms which is based on antibodies. The mammalian immune system operates by recognising all possible chemical shapes (epitopes), classifying them as self or non-self, and then generating systems to control any organism which bears foreign epitopes. An important part of the effector arm of the immune system is antibody which can recognise and combine with foreign epitopes. One advantage of antibody over antibiotics is that resistance cannot emerge. If the bacteria acquire new epitopes the immune system can respond with new antibody.

There are five major categories of immunoglobulin antibody i.e. $\operatorname{IgG}, \operatorname{Ig} A, \operatorname{IgM}, \operatorname{IgD}$ and $\operatorname{IgE}$. The antibody in the highest concentration in the systemic circulation is IgG and this protects against invasive infection. $\operatorname{IgA}$ is the major antibody in surface secretions. The IgA molecule is a dimer held together by a junctional polypeptide chain. It also has a second polypeptide chain, called a secretory piece, which protects the molecule against proteolytic breakdown in the gastro-intestinal tract.

Many bacteria, fungi and protozoa which cause human disease normally live on the body surface. The major sites are the mucosa of the gastro-intestinal tract, the mucosa of the respiratory tract and the skin. These organisms can cause local tissue injury and inflammation or can invade the body to cause systemic disease. In the respiratory tract and the gastrointestinal tract infection is prevented by the mucosal immune system which generates and secretes IgA antibodies onto the mucosal surface. These antibodies recognise and combine with epitopes on the surface of micro-organisms and prevent their adherence to the mucosal surface. This limits their ability to cause local damage and prevents invasion of host tissue. The total amount of IgA secreted each day by the mucosal immune system onto the body surface is greater than the amount of IgG produced internally. This indicates the relative importance of $\mathrm{IgA}$ in preventing disease.

IgA molecules are present in saliva, milk and the mucus secretions of the gastro-intestinal and respiratory tracts. $\operatorname{Ig} \mathrm{A}$ is the major immunoglobulin in human milk and there is considerable evidence that human milk protects the infant against a range of infections in the first few months of life. IgA is also present in the milk of other mammals including ruminants such as cows, sheep and goats. In ruminants, however, there is no transplacental transfer of IgG as seen in humans. Instead colostrum in the first few days following parturition contains high levels of both $\mathrm{IgG}$ and $\mathrm{IgA}$ as well as other substances which are important in immune defence. The calf absorbs the immunoglobulins into the systemic circulation and they are used for internal defence. Subsequently post colostrum milk contains both $\operatorname{IgG}$ and $\operatorname{IgA}$ but absorption is reduced and these antibodies are used for surface defence.

The invention is the use of post colostrum mammalian milk with high titres of $\operatorname{IgA}$ antibodies to specific epitopes to treat surface infections by microorganisms which carry the specific epitopes.

\section{H PYLORI}

$H$ pylori is an important and common human pathogen. It is present on the gastric mucosal surface of $25 \%$ of the population in the United Kingdom. Poor social conditions are associated with increased prevalence and carriage of the organism is more common in many less technologically advanced countries. $H$ pylori causes inflammation of the gastric mucosa leading to gastric and duodenal ulceration. It also greatly increases the risk of gastric cancer. We have found that there are $\operatorname{IgA}$ antibodies in post colostrum bovine milk which specifically recognise epitopes of $H$ pylori. The titres vary markedly between individual animals with very high levels in just a few animals. Our experiments indicate that the IgA antibodies to $H$ pylori cross react with epitopes on the surface of campylobacter organisms, which are common farmyard pathogens. It appears likely that natural infection by campylobacter has led to the production of $\operatorname{IgA}$ antibodies which recognise $H$ pylori.

A specific example of the use of our invention is the treatment of $H$ pylori gastric infection using milk from cows, or from other mammals, with high titres of $\operatorname{IgA}$ antibody to $H$ pylori. The milk could be used alone or in combination with established treatments to increase the chance of cure. 
In order to raise the titre of specific $\mathrm{IgA}$ antibodies in milk we intend to immunise cows, and other mammals including sheep, goats and pigs, using $H$ pylori antigens. Our aim is to produce high titres of $\operatorname{IgA}$, not other immunoglobulins, and we will induce mucosal immunity by exposing the mucosa of the upper respiratory tract to killed $H$ pylori organisms in association with adjuvants such as modified cholera toxin Those familiar with the art will know that this is a standard procedure for inducing mucosal immunity and producing high titres of $\operatorname{IgA}$.

Milk is a complex mixture of chemicals, optimally designed by the process of evolution, for nutrition and for defence against infection. There are a number of other natural antibacterial substances which augment the action of $\operatorname{IgA}$ and it is our intention to use milk in as pure a form as possible in order to exploit this natural synergy. In using milk as therapy, however, we must ensure there is no risk of passing infection from milk to humans. We will avoid pasteurisation as this can impair natural defences but we will use filtration to reduce contaminating micro-organisms. In addition systems of preservation will be used including freeze drying.

\section{VEROTOXIN PRODUCING E COLI}

Another specific example of the use of the invention is in the treatment of infection by verotoxin producing $E$ coli. These bacteria grow in the gastrointestinal tract and produce potent toxins which are absorbed into the circulation and cause systemic damage. Infection by this organism is an increasing problem and there have been several large outbreaks of disease with considerable morbidity and mortality. A major problem in dealing with this disease is that the use of antibiotics can cause bacterial cell death and release of verotoxins leading to an exacerbation of the disease. Therapy is limited to trying to reduce the concentration of circulating toxins by systems such as haemofiltration.

The therapeutic use of mammalian milk with high titre IgA antibodies to verotoxin would prevent verotoxin produced in the intestine entering the circulation. This treatment could be used alone or in combination with antibiotics to treat the disease.
The production of high titre $\operatorname{IgA}$ in mammalian milk directed against verotoxin will follow the same general principles as in the case of $H$ pylori. Initially cows will be selected for study, those with naturally raised titres of $\operatorname{IgA}$ in milk will be detected using ELISA techniques, these animals will then be immunised to raise the titre even further and the milk will then be used for therapy. Subsequently other mammals will be investigated including sheep, goats and pigs.

\section{Claims}

1 A method of treating and preventing surface infections in mammals by micro-organisms using post colostrum mammalian milk with high titres of $\operatorname{IgA}$ antibodies which recognise epitopes on the infecting organisms.

2 The surface infections in claim 1 include infection by bacteria, fungi and protozoa involving the skin, the mucosa of the respiratory tract, the mucosa of the gastro-intestinal tract and the vagina.

3 The therapy in claim 1 will be taken orally or applied directly to the relevant surface.

4 High titres of specific IgA as stated in claim 1 will be produced by standard techniques of inducing mucosal immunity involving introducing killed organisms to the surface of the upper respiratory tract in association with a standard adjuvant.

5 The therapy in claim 1 will use whole milk after filtration to reduce the possibility of contamination by microorganisms.

6 The therapy in claim 1 will be used fresh or will be reconstituted after freeze drying.

7 The therapy in claim 1 will be used alone or in combination with other recognised therapies for the specific microbial infection.

\section{New Undergraduate MBMJ Prize}

The Leese Bequest has kindly agreed to contribute to or fund a prize of $£ 200$ relating to the fields of respiratory or cardiovascular medicine. The details have yet to be finalised and more information will be available in the next issue. 